\title{
Mechanistic Dissection of Spatial Organization in NF-кB Signaling Pathways by Hybrid Simulations
}

\author{
Yinghao $\mathrm{Wu}^{1 *}$, Kalyani Dhusia ${ }^{1}$, Zhaoqian $\mathrm{Su}^{1}$ \\ ${ }^{1}$ Department of Systems and Computational Biology, Albert Einstein College of \\ Medicine, 1300 Morris Park Avenue, Bronx, NY, 10461
}

*Corresponding authors:

Yinghao $\mathrm{Wu}$

Phone: (718) 678-1232, Fax: (718) 678-1018, E-mail:yinghao.wu@einsteinmed.org 


\section{ABSTRACT}

The nuclear factor kappa-light-chain-enhancer of activated B cells $(\mathrm{NF}-\kappa \mathrm{B})$ is one of the most important transcription factors involved in the regulation of inflammatory signaling pathways. Inappropriate activation of these pathways has been linked to autoimmunity and cancers. Emerging experimental evidences have been showing the existence of elaborate spatial organizations for various molecular components in the pathways. One example is the scaffold protein tumor necrosis factor receptor associated factor (TRAF). While most TRAF proteins form trimeric quaternary structure through their coiled-coil regions, the N-terminal region of some members in the family can further be dimerized. This dimerization of TRAF trimers can drive them into higher-order clusters as a response to receptor stimulation, which functions as a spatial platform to mediate the downstream poly-ubiquitination. However, the molecular mechanism underlying the TRAF protein clustering and its functional impacts are not well-understood. In this article, we developed a hybrid simulation method to tackle this problem. The assembly of TRAFbased signaling platform at the membrane-proximal region is modeled with spatial resolution, while the dynamics of downstream signaling network, including the negative feedbacks through various signaling inhibitors, is simulated as stochastic chemical reactions. These two algorithms are further synchronized under a multiscale simulation framework. Using this computational model, we illustrated that the formation of TRAF signaling platform can trigger an oscillatory $\mathrm{NF}-\kappa \mathrm{B}$ response. We further demonstrated that the temporal patterns of downstream signal oscillations are closely regulated by the spatial factors of TRAF clustering, such as the geometry and energy of dimerization between TRAF trimers. In general, our study sheds light on the basic mechanism of NF$\mathrm{\kappa B}$ signaling pathway and highlights the functional importance of spatial regulation within the pathway. The simulation framework also showcases its potential of application to other signaling pathways in cells. 


\section{Introduction}

The innate immune system constitutes the first line of host defense during infections by invading pathogens [1]. This defensive response, called inflammation, is a complicated process orchestrated by many different cellular components [2, 3]. At the onset of inflammation, cytokines are released from immune cells such as macrophage after they capture infected cells [2]. The nuclear factor kappa-light-chain-enhancer of activated $\mathrm{B}$ cells $(\mathrm{NF}-\mathrm{\kappa B})$ is a critical transcription factor regulates the expressions of cytokines that stimulate inflammatory responses $[4,5]$. Under normal condition, NF- $\kappa B$ is

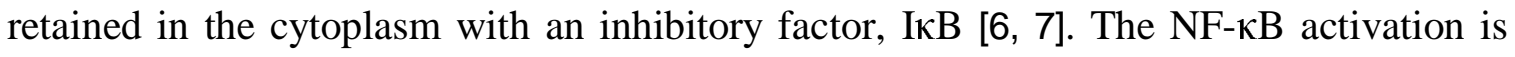
started from the binding of membrane receptors, mainly in tumor necrosis factor receptor (TNFR) superfamily to their extracellular ligands [8]. The ligand binding of receptors triggers the recruitment of adaptor proteins, such as TNF receptor associated factor (TRAF), to their cytoplasmic domains [9]. While the C-terminal domain of TRAF maintains interactions with upstream receptors, the N-terminal regions function as platform to mediate the process of poly-ubiquitination [10]. The ubiquitination leads to the degradation of I $\mathrm{B}$, so that NF- $\kappa \mathrm{B}$ can be released and enter cell nucleus to initiate gene expression [11, 12]. It has been found that spatial organizations of proteins are highly involved in signaling pathways $[13,14]$. Such phenomena have also been observed in $\mathrm{NF}-\kappa \mathrm{B}$ signaling pathway [15]. For instance, in vivo experiments have shown that TRAF proteins can aggregate to higher-order spatial patterns as a response to receptor stimulation $[16,17]$. The structural evidences further indicate that the $\mathrm{N}$-terminal region in some TRAF proteins, such as TRAF6 and TRAF2, is dimeric [16, 18, 19]. The capability of high-order pattern formation can be abolished by a mutant that disables this dimerization. As a result, it was proposed that the dimerization can lead to a twodimensional clustering of trimeric TRAF protein complexes. However, it is not fully understood how this spatial organization regulates the dynamics in NF- $\kappa \mathrm{B}$ signaling pathway.

Due to the functional importance of $\mathrm{NF}-\kappa \mathrm{B}$ in immunity, the molecular mechanisms of its signaling pathway are under intensive investigation [20]. Comparing with other traditional wet-lab experiments, computational modeling is more convenient to 
explore the complexity of a biological system on a mechanistic level. As a result, a large variety of models have been developed on different scales of the pathway. The original model focused on the role of $\mathrm{I} \kappa \mathrm{B}$ in regulating the temporal dynamics of NF- $\kappa \mathrm{B}$ by using a set of ordinary differential equations (ODE) [21, 22]. More recently, stochastic simulations and agent-based modeling (ABM) approaches were applied to generate new hypotheses on the behavior of various molecular components in the NF- $\mathrm{B}$ pathway [2326]. However, information about spatial organization of these components, such as the oligomerization of TRAF proteins, has not been incorporated into these models. On the other hand, methods in another class of simulation technique, including MCELL [27], Smoldyn [28, 29], and more recently SpringSaLaD [30, 31], use lattice-based or particlebased models to implement the diffusions of biomolecules within a more realistic cellular environment. They can be used to simulate the formation of spatial patterns on the subcellular level, such as protein complex assembly or phase separation [32-40]. Unfortunately, these methods are intractable to be applied to study the dynamics of an entire signaling pathway because of their high demands for computational resources. Overall, it is highly challenging to develop a simulation method which is able to capture a signaling event with both spatial resolution and its functional impacts on the whole signaling networks.

In order to overcome this challenge, a hybrid simulation approach is presented to describe the dynamics in the NF- $\kappa \mathrm{B}$ signaling pathway. The method contains two coupled systems. The assembly of TRAF-based signaling platform at the membraneproximal region is modeled by a rigid-body-based diffusion-reaction algorithm, while the downstream signaling events, including the recruitment of I $\mathrm{B}$ kinase (IKK), phosphorylation of I $\kappa \mathrm{B}$ and activation of NF- $\kappa \mathrm{B}$ are simulated as stochastic chemical reactions. These two levels of simulations are integrated together, so that how spatial variations in the signaling platform affect the rest of the pathway can be quantitatively analyzed. The signaling network is further regulated by negative feedbacks through different inhibitors such as IkB and A20, which leads to an oscillatory NF- $\mathrm{kB}$ response. We demonstrated that the temporal patterns of downstream signal oscillations are closely correlated with the spatial organization of upstream platform assembly. Our simulation results also suggested that the cellular dynamics of NF- $\mathrm{kB}$ signaling pathway can be fine- 
tuned by a single pair of molecular interaction between TRAF proteins. Altogether, this study sheds light on the basic mechanism of NF- $\mathrm{kB}$ signaling pathway and highlights the functional importance of spatial regulation. The hybrid simulation framework also showcases its potential of application to other systems of cell signaling pathways.

\section{Results and Discussions}

\section{General description of the outputs from simulations of the signaling network}

Most members in TRAF family contain a RING domain at their N-terminus, a TRAF-C domain with seven to eight anti-parallel $\beta$-strand folds at their C-terminus, and a coiled-coil region in the middle [41]. While the C-terminal domain of TRAF maintains interactions with upstream receptors, the $\mathrm{N}$-terminal regions function as platform to mediate the downstream poly-ubiquitination, as well as the cis-interactions between themselves [18]. Moreover, most members in TRAF family form trimeric quaternary structure through their coiled-coil regions [42]. We are mainly focusing on the spatial clustering of TRAF trimers and its impacts on regulating the downstream signaling dynamics. The formation of upstream ligand-receptor complex is beyond the scope of this study. As a result, we assume that the ligand-receptor complexes have already preformed on cell surface before our simulations and the trimeric scaffold proteins have already bound to the cytoplasmic domains of receptors (Figure 1a). Based on this assumption, the TRAF trimer and the ligand-receptor complex are modeled as one single rigid body attached to the cell membrane. Each rigid body contains three binding sites to maintain the $c$ is-interaction between TRAF trimers. The movements of each TRAF trimer are confined within the two-dimensional membrane proximal area. The multiple cisinteractions involved in each TRAF trimer and its two-dimensional movements can further result in the higher-order clustering, which is simulated by a diffusion-reaction algorithm. Detailed model representation of TRAF trimers and the follow-up algorithm used to simulate their clustering are described in the Methods.

In addition to form cis-interactions, the N-terminal RING domain of TRAF protein also functions as an E3 ubiquitin ligase. It recruits the E2 ubiquitin-conjugating enzyme such as Ubc13 so that the poly-ubiquitin chains can be formed [19]. The 
formation of a cis-interaction between two TRAF trimers thus facilitates the assembly of linear ubiquitin chain assembly complex (LUBAC) [43]. The LUBAC further provide the scaffold to enable the activation of the kinases IKK. Upon activation, IKK can induce the phosphorylation of I $\mathrm{KB}$, which forms a complex with the transcription factor NF- $\kappa \mathrm{B}$ as an inhibitor [11]. The complex will be dissociated after the phosphorylation of IкB, so that the transcription factor can be freely released. The phosphorylated inhibitor later undergoes ubiquitin-dependent proteolysis, while the released transcription factor enters cell nucleus and bind to the promoter and turn on its target genes. The genetic regulation of two proteins is specific modeled in the network. The first is the inhibitor IkB itself.

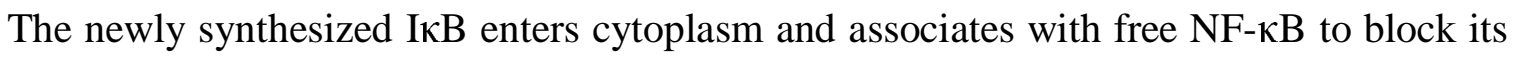
function as a transcription regulator [44]. The second protein which gene is also turned on by NF- $\kappa B$ is $\mathrm{A}_{20}$. Upon synthesis, the protein also functions as an inhibitor to diminish the activation process of IKK [45]. The diagram of the network is shown in Figure 1b, while its mathematical description is presented in the Method.

Based on the mathematical representation of all reactions in the signaling network, the dynamics of the system is simulated stochastically by Gillespie algorithm. The signaling network has further been coupled with the spatial clustering of TRAF trimers under a hybrid simulation framework. The specific procedure of the hybrid simulation which combined Gillespie algorithm with the spatial model of TRAF clustering is delineated in the Method. The results from this hybrid simulation are summarized in Figure 2. Specifically, 200 TRAF trimers were randomly placed in the two-dimensional membrane proximal region as an initial configuration (Figure 2a). The length of each side in this square region is $1000 \mathrm{~nm}$, along both $\mathrm{X}$ and $\mathrm{Y}$ directions, which gives a total area of $1 \mu \mathrm{m}^{2}$. Some representative snapshots of the clustering process are also plotted along the simulation trajectory. We found that TRAF trimers start to aggregate into small oligomers (Figure 2b). These oligomers are organized into hexagonal lattice based on the spatial symmetry of three binding sites in each trimer. The formation of these oligomer is a very dynamic process. TRAF trimers constantly left one oligomer and joined another one. As a result, small oligomers either disappeared or merged into neighboring larger oligomers, leading into a configuration in which the number of clusters became smaller and their sizes continued growing, as shown in Figure 2c. Ultimately, most trimers 
aggregated together into a final larger cluster and the system reached equilibrium, as shown in Figure 2d.

The number of cis-interactions formed between TRAF trimers was further plotted in Figure 2e as a function of simulation time steps. The figure shows that the number of cis-interactions increased very fast at the beginning of the simulation, indicating an initial seeding process. The increase of cis-interactions became slower as the oligomers in the system grew, and finally reached saturation after $4.5 \times 10^{5}$ simulation time steps, corresponding to the formation of the final cluster. The kinetic profiles of molecules in the downstream signaling network were further plotted in the following figures. Specifically, the numbers of NF- $\kappa$ B molecules which bind to I $\kappa$ B and DNA are shown in blue and orange curves in Figure 2.f, respectively. A periodic boost of DNA-bound NF$\kappa \mathrm{B}$ is displayed in the figure, corresponding to the timing of dissociation between NF- $\kappa \mathrm{B}$

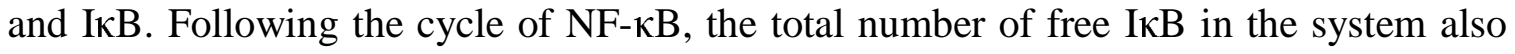
oscillated along the simulation, as illustrated in Figure 2.g. This oscillatory behavior is the result of the negative feedback regulation in the network, which is implemented by $\mathrm{I} \kappa \mathrm{B}$ and $\mathrm{A}_{20}$. Additionally, we found that the oscillation was initiated after the number of total cis-interactions between TRAF trimers almost stop changing, indicating that the formation of large clusters is critical to activate the downstream signaling pathway. In

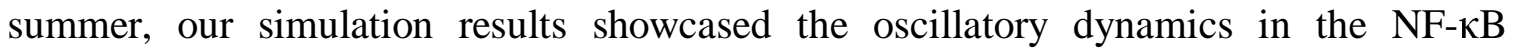
signaling pathway and its correlation with the spatial organization of molecular components in the pathway.

\section{The comparison of signaling dynamics in systems with and without TRAF clustering}

In order to further elucidate the functional importance of TRAF clustering in regulation of downstream signaling pathway, we carried out two separate simulations with different scenarios. The TRAF trimers in the first scenario, as we descried before, contain three binding sites. As a result, each trimer can simultaneously form cisinteractions with three structural neighbors, leading into the formation of a highly ordered spatial pattern. In contrast, a control system was designed in the second scenario. In this control system, we disabled the possibility of high-order clustering among TRAF proteins. 
This was achieved by assigning only one binding site to each TRAF protein instead of three, so that only cis-dimers can be formed between two proteins. As the initial configuration of the first scenario, 200 rigid bodies of TRAF trimers were randomly placed on a two-dimensional square surface with an area of $1000 \times 1000 \mathrm{~nm}^{2}$. On the other hand, the control simulation contains 600 rigid bodies randomly distributed on the surface with the same area to maintain the same level of possible total interactions as in the first scenario. All the other parameters in the diffusion-reaction simulation of control scenario such as diffusion and binding constants remain unchanged. The binding affinity of cis-interactions in both systems equals -10kT. Moreover, in the stochastic simulations of downstream signaling network, the same values of rate constants were used for the second scenario as for the first scenario.

The comparison of simulation results between these two scenarios are summarized in Figure 3. The total number of cis-interactions formed between TRAF trimers along the simulation in the first system is plotted by the red curve in Figure 3.a, while the total number of dimerized cis-interactions formed in the control system is plotted by the red curve. The figure shows that the number of cis-interactions in the system which can form clusters grew more slowly but reached a much higher level than the system which can only form dimers, although the binding affinity and total binding sites in both systems are the same. Moreover, the level of fluctuations in the first system is also lower than the second one. The final configurations at the end of two simulations are compared with each other in Figure 3c and Figure 3d, respectively. Different from a small number of large clusters oligomerized in the first system, a large number of dimers are randomly distributed in the control system.

In order to quantitatively estimate the statistical significance of observed differences between these two systems, a two-sample student's t-test was carried out to the data collected from these two trajectories, which distributions are plotted as histograms in Figure 3.b. In detail, the average number of cis-interactions in the distribution of dimerized system is 75.51 and its standard deviation equals 7.16. Relatively, the average number of cis-interactions in the distribution of oligomerized system is 182.53 and its standard deviation equals 3.04. The null hypothesis is that no 
difference exists between two distributions. It was tested at a 95\% confidence interval. The calculated t-score equals 650.28 with the P-value lower than 0.0001. This small Pvalue from the t-test indicates that the null hypothesis can be rejected and the alternative hypothesis can be accepted, i.e., the spatial arrangement of TRAF oligomers lead into significant change in the level of cis-interactions comparing with the system in which oligomers are not allowed to form.

The outputs from the downstream signaling networks of the two comparative systems are shown in the following panels of the figure. The changes of NF- $\kappa \mathrm{B}$ that bound to IאB or DNA in the first system are plotted as a function of the simulation time with blue and yellow curves in Figure 3e. The oscillations were observed in the system, in which the number of NF- $\mathrm{B} / \mathrm{DNA}$ complexes was boosted periodically after the clusters of TRAF trimers were stabilized, corresponding to the red curve in Figure 3a. The impulse of NF- $\kappa \mathrm{B} / \mathrm{DNA}$ complexes is coincident with the drop of NF- $\kappa \mathrm{B} / \mathrm{I} \kappa \mathrm{B}$ complexes, which is due to the phosphorylation of I $\mathrm{KB}$ by activated IKK. This oscillatory dynamic of components in the network indicates that the signaling pathway was turned on by the clustering of TRAF trimers. In contrast, the number of NF- $\kappa \mathrm{B} / \mathrm{I} \kappa \mathrm{B}$ complexes remained at its initial level throughout the simulation in the second system. As a result, no NF- $\kappa \mathrm{B} / \mathrm{DNA}$ complex was obtained before the end of the simulation, corresponding to the curves plotted in Figure 3f. This result suggests that the signaling pathway could not be effectively turned on due to the less numbers and higher fluctuations of cis-dimers formed in the control scenario.

In summary, this comparative study demonstrated the possibility that systems with the same sets of rate parameters can evolve into very different temporal dynamics, only because of the different spatial organizations of signaling molecules. More specifically, a threshold-like behavior is controlled by the geometric arrangement of cisinteraction between TRAF scaffold proteins. Multiple binding sites in TRAF trimers lead to their high-order aggregation at membrane proximal region. Comparing with the regular dimer, each trimer in a cluster is simultaneously involved in three cis-interactions with its neighbor. These interlocked systems are kinetically more difficult to be dissociated. Consequently, they provide stable platform to downstream signal activation. Without this 
trimeric quaternary structure, on the other hand, TRAF proteins can only dimerized. Comparing with the highly ordered oligomers, these dimers are easier to be dissociated, although the binding affinity of the cis-interaction remains unchanged. As a result, the poly-ubiquitination facilitated by dimerized TRAF proteins alone cannot pass the threshold of downstream signal activation.

\section{Change the binding constants in the oligomerization}

We have estimated the importance of TRAF trimers' multiple cis-binding sites not only in their assembly into higher-order structures, but also in the initiation of NF-кB signaling pathway. In this section, we further explore the energetic impacts of this cisinteraction on spatial-temporal dynamics of the system. Specifically, the binding affinity for a given pair of binding sites between two TRAF trimers was charged to different values in the diffusion-reaction simulation. Three scenarios were tested separately. A weak binding affinity (-6kT) was used in the first scenario. In comparison, a strong binding affinity (-14kT) was used in the second scenario. In addition to these two systems, a moderate value of binding affinity $(-8 \mathrm{kT})$ was also tested as the third scenario. All the other parameters in both TRAF clustering section and signaling network section of the simulations are the same. The kinetic profiles generated from the simulations of these three systems are plotted in Figure 4.

The numbers of cis-interactions formed in the three scenarios are plotted in Figure $4 \mathbf{a}$ as a function of simulation time steps. The profile with the weak binding affinity is shown by the black curve, which indicates a low number of cis-interactions. On the other hand, a much higher level of cis-interactions were derived in the system with strong bind affinity, as shown by the blue curve. A more interesting kinetic profile was observed in the system with moderate binding affinity, corresponding to the red curve in Figure 4a. The figure suggests that a small reduce of affinity from $-6 \mathrm{kT}$ to $-8 \mathrm{kT}$ significantly increased the level of cis-interactions. Surprisingly, the number of total interactions formed by the end of the simulation with moderate binding affinity is more than 200, even higher than the number found in the system with much stronger cisinteractions. Moreover, comparing with the system of strong affinity which reached 
equilibrium at the very early stage of simulation, the number of cis-interactions in the system of moderate binding affinity increased much slower.

The dynamics in the downstream signaling networks of three systems are further shown in Figure $\mathbf{4 b}$. The changes of free IKB are plotted as a function of simulation time steps. An oscillation on the level of IкB was observed in the system of moderate binding affinity, as shown by the red curve in the figure. The oscillation was started when the stimulation time reached $1 \times 10^{5}$ steps, consistent with the stage as the number of cisinteractions in the system didn't further increase (shown by the red curve in Figure 4a). In contrast, no free I $\kappa \mathrm{B}$ was observed in the system with weak binding affinity. This is due to the low level of cis-interactions formed in the system. The poly-ubiquitin chains recruited by this small number of cis-interaction between TRAF proteins were not enough to active IKK in the system, and prevented the IKB from being phosphorylated. As a result, the genes regulated by $\mathrm{NF}-\kappa \mathrm{B}$ cannot be turned on throughout the simulation. It is worth mentioning that no oscillation of free IкB was also obtained in the system with strong binding affinity. The I $\mathrm{B}$ B was produced at very late stage of the simulation after the time passed $4 \times 10^{5}$ steps. However, it reached to a very low level, but then start to decay all the way through the end of the simulation, as shown by the blue curve in Figure 4b. The results from the simulations of these three systems therefore suggest that the oscillatory patterns of the signaling dynamics are very sensitive to the strength of cisinteraction between TRAF proteins.

In order to test the correlation between the oscillatory patterns of the signaling pathway and the binding affinity of the cis-interaction on a more statistically meaningful level, systematical tests were carried out to systems which binding affinities of cisinteraction were ranged from $-6 \mathrm{kT}$ to $-15 \mathrm{kT}$ with an interval of $1 \mathrm{kT}$. Multiple trajectories were generated for each system, while a longer simulation time $\left(2 \times 10^{6}\right.$ steps $)$ was given for each trajectory. At the end of all simulations, we counted the number of oscillatory periods observed in each trajectory. The average number of oscillatory periods was then calculated for each system depending on the values of binding affinity. The calculated results are summarized as a histogram in Figure 4c. The figure confirms that oscillations were not observed under weak binding affinities, but suddenly appeared when affinities 
are below $-7 \mathrm{kT}$, suggesting that there is phase transition in the system. Further enhancement of binding affinity doesn't change the frequency of oscillation too much until it becomes stronger than $-12 \mathrm{kT}$. Above this region, the oscillatory frequency will be gradually reduced if the cis-binding affinity increases. Finally, no more oscillation exists after the binding affinity equals $-15 \mathrm{kT}$. This is consistent with the signaling outputs illustrated in Figure $\mathbf{4 b}$.

To further understand how NF- $\kappa \mathrm{B}$ signals can be spatially regulated by TRAF protein clustering, the final configurations from some of the simulation systems were plotted in Figure 5. The final configurations from the systems which binding affinities equal $-7 \mathrm{kT}$ and $-8 \mathrm{kT}$ are shown in Figure 5a and Figure 5b, respectively. With only a slight change in the binding affinity, very different spatial pattern was derived in the figures. This difference can be explained by the phase transition of TRAF clustering which drives the system from a scattered distribution of trimers to their condensation into a single cluster. The network of trimers formed within the cluster stabilizes the cisinteractions and thus facilitate the signal activation of downstream pathway. In comparison, the final configurations from two systems selected from the other end of the binding affinity are shown in Figure 5c and Figure 5d. These figures show that systems with stronger binding affinity contain more clusters with smaller sizes. Comparing with the signal big cluster formed in the system with binding affinity of $-8 \mathrm{kT}$, five clusters were obtained in the system with binding affinity of $-13 \mathrm{kT}$ and each cluster on average contains 40 trimers, as shown in Figure 5c. When the binding affinity reaches $-15 \mathrm{kT}$, as shown in Figure 5d, the number of clusters in the system increases to 20 and each cluster on average only contains 10 TRAF trimers. These results suggest that the strong binding affinities kinetically trap TRAF trimers into small clusters, which plays negative role in activating the oscillations in the downstream signaling network.

Taken together, our simulation results show that the increase of cis-binding affinity triggers the phase transition of TRAF clustering. This phase transition results in a threshold-like signal response from the downstream pathway. Moreover, the large-scale TRAF clusters is kinetically slow to assemble, but highly stable after its formation. This slow kinetics of the assembling process and high stability of the clustered structure assure 
that the signaling pathway only responses to a persistent and high dose of extracellular stimulations, therefore promote the fidelity of signal transduction within stochastic cellular environments [46]. Finally, the oscillation of NF-- $\mathrm{BB}$ signals can only be archived through a very dynamic process by cis-interactions with moderate binding affinities. The TRAF proteins with overly strong cis-interactions will be kinetically trapped in small clusters, and further impede their function in activating the downstream signal oscillation. This suggests that the molecular interactions in the NF- $\kappa \mathrm{B}$ signaling pathway are tuned within a specific range by natural selection to maintain its appropriate functions.

\section{Conclusions}

NF- $\kappa \mathrm{B}$ signaling pathway is one of the most important cell signaling pathways involved in inflammatory responses [47]. Recent experimental evidences started to show that signal transduction in the pathway is spatially modulated by its different molecular components [48]. However, the molecular mechanism of these spatial regulation and their functional impacts are not well understood. In order to tackle this problem, we developed a hybrid model in which the NF- $\kappa \mathrm{B}$ signaling pathway is decomposed into two simulation scenarios. The physical process of TRAF clustering at membrane proximal region is simulated by a rigid-body-based diffusion-reaction algorithm, while the downstream signaling network is simulated by stochastic simulation with Gillespie algorithm. These two algorithms are further synchronized under a multiscale simulation framework. Using this simulation method, we illustrated that the formation of TRAFmediated 2D signaling platform is a critical factor to regulate the downstream oscillatory dynamics in the signaling network. The modification of cis-interaction between TRAF proteins leads to the changes of their clustering patterns at membrane proximal regions, and further affects the NF- $\mathrm{kB}$ response. Interestingly, our results show that mutations either weaken or strengthen this cis-interaction can cause the abolishment of oscillation in the pathway. This observation suggests that molecular elements and their interactions in a signaling network are elaborately designed to carried out their appropriate functions. In summary, the hybrid simulation developed in this study shows possibility to model a signaling system with both spatial resolution and functional implication. The results from 
the simulations provides the general biological insights to the interplay between the spatial assembly of individual signaling molecules and the threshold-like output from the entire signaling network, which offers a potentially new way to control signaling pathways in cellular systems.

\section{Methods}

\section{Mathematical representation of the signaling network}

Following the spatial assembly of TRAF signaling platform, the changes of population for each type of molecular components in the downstream signaling network are quantitatively described by a set of ordinary differential equations (ODE). Specifically, the numbers of active IKK and inactive IKK in the system at time $t$ are changed by solving the following two equations.

$$
\begin{aligned}
& \frac{d[a \cdot I K K(t)]}{d t}=-r_{1}[a \cdot I K K(t)]+r_{2}[i \cdot I K K(t)] \times \frac{[\operatorname{LUBAC}(t)]^{N}}{[\operatorname{LUBAC}(t)]^{N}+\left[K_{2} \times\left(1+\left[A_{20}(t)\right] / K_{I}\right)\right]^{N}} \\
& \frac{d[i \cdot I K K(t)]}{d t}=r_{1}[a \cdot I K K(t)]-r_{2}[i \cdot I K K(t)] \times \frac{[\operatorname{LUBAC}(t)]^{N}}{[\operatorname{LUBAC}(t)]^{N}+\left[K_{2} \times\left(1+\left[A_{20}(t)\right] / K_{I}\right)\right]^{N}}
\end{aligned}
$$

In above equations, $[a \cdot I K K(t)]$ and $[i \cdot I K K(t)]$ are the numbers of active IKK and inactive IKK at time $t$, respectively. The first term on the right-hand side of equation (1) represents the transition of IKK from its active form to inactive form, where the parameter $r_{l}$ indicates the rate of the transition. The second term on the right-hand side of equation (1) represents the transition of IKK from its inactive form to active form. This reaction is catalyzed by the upstream poly-ubiquitin chains LUBAC, in which $r_{2}$ and $K_{2}$ are the maximal rate and saturation coefficient in the catalysis. The number of polyubiquitin chains in the system $[L U B A C(t)]$ is determined by the number of cisinteractions between TRAF trimers in the upstream signaling platform. Moreover, considering that LUBAC itself is a highly ordered signaling machinery, a Hill coefficient [49] $N$ is used to model the cooperativity in its assembly and its recruitment of IKK. 
Finally, the catalysis of this transition reaction is also restrained by protein $\mathrm{A}_{20}$ as a competitive inhibitor. The efficiency of inhibition is controlled by the concentration of $\mathrm{A}_{20}$ and the parameter $K_{I}$.

The activated IKK kinase further phosphorylates the inhibitory IкB subunit in the $\mathrm{I} \kappa \mathrm{B} / N F-\kappa B$ complex. The number of phosphorylated complexes in the system at time $t$ can be changed by solving the following equation.

$$
\frac{d[I \kappa B \cdot P / N F-\kappa B(t)]}{d t}=-r_{4}[I \kappa B \cdot P / N F-\kappa B(t)]+\frac{r_{3}[a \cdot I K K(t)][I \kappa B / N F-\kappa B(t)]}{[I \kappa B / N F-\kappa B(t)]+K_{3}}
$$

In above equation, $[I \kappa B / N F-\kappa B(t)]$ and $[I \kappa B \cdot P / N F-\kappa B(t)]$ are the numbers of phosphorylated and un-phosphorylated complex at time $t$, respectively. The second term on the right-hand side of equation (3) describes the phosphorylation process, which rate depends on the concentration of activated IKK [a.IKK(t)]. The parameters $r_{3}$ and $K_{3}$ in the reaction are the maximal rate and saturation coefficient in the phosphorylation as described by Michaelis-Menten kinetics. The first term on the right-hand side of equation (3) describes the dissociation reaction of IкB/NF- $\kappa B$ complex due to the phosphorylation of IKB, in which $r_{4}$ represents the rate constant of dissociation.

On the other hand, the number of unphosphorylated complex in the system is decreased by the IKK induced phosphorylation, but increased by the association reaction

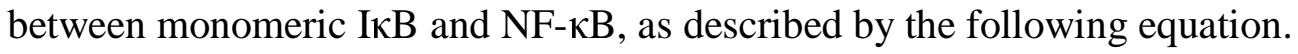

$$
\frac{d[I \kappa B / N F-\kappa B(t)]}{d t}=-\frac{r_{3}[a \cdot I K K(t)][I \kappa B / N F-\kappa B(t)]}{[I \kappa B / N F-\kappa B(t)]+K_{3}}+r_{6}[I \kappa B(t)][N F-\kappa B(t)]
$$

The parameter $r_{6}$ in equation (4) is defined as the rate constant which regulates the

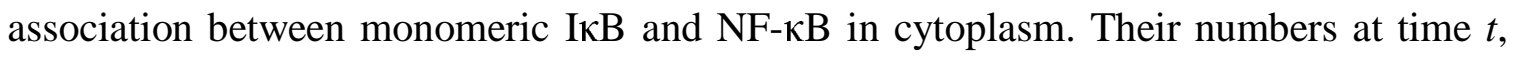
$[I \kappa B(t)]$ and $[N F-\kappa B(t)]$ are changed by the following two equations:

$$
\frac{d[I \kappa B(t)]}{d t}=\frac{s_{7}[N F-\kappa B \cdot D N A(t-\tau)]}{[N F-\kappa B \cdot D N A(t-\tau)]+K_{7}}-r_{6}[I \kappa B(t)][N F-\kappa B(t)]
$$




$$
\begin{gathered}
\frac{d[N F-\kappa B(t)]}{d t}=r_{4}[I \kappa B \cdot P / N F-\kappa B(t)]-r_{6}[I \kappa B(t)][N F-\kappa B(t)] \\
+r_{10}[N F-\kappa B \cdot D N A(t)]-r_{9}[N F-\kappa B(t)]
\end{gathered}
$$

The second term on the right-hand side of equation (5) delineates the dissociation of $\mathrm{I} \kappa \mathrm{B} / \mathrm{NF}-\kappa \mathrm{B}$ complex, as described above. Additionally, the dissociation of NF- $\kappa \mathrm{B}$ from phosphorylated IкB enters cell nucleus and binds to its targeted DNA sequence as a transcription factor. The formation of NF- $\kappa \mathrm{B} \cdot \mathrm{DNA}$ complex regulates the expression of specific genes, including its own inhibitor $\mathrm{I} \kappa \mathrm{B}$, which corresponds to the first term on the right-hand side equation (5). We introduced the constant $\tau$ in the reaction to describe the

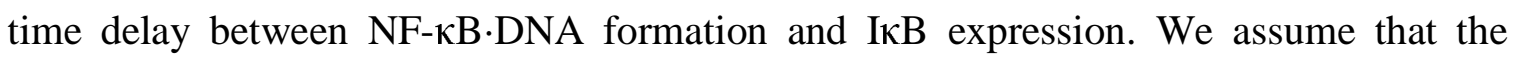
expression rate of $\mathrm{I} \kappa \mathrm{B}$ depends on the concentration of activated transcriptional factors $[N F-\kappa B \cdot D N A(t-\tau)]$ and reaches a maximum value $s_{7}$. The parameter $K_{7}$ is the saturation coefficient in the regulation of protein synthesis as described by Michaelis-Menten kinetics. In equation (6), on the other hand, the first two terms on its right-hand side have been introduced above, while the next two terms described the dynamic process in which $\mathrm{NF}-\kappa \mathrm{B}$ is translocated from cytoplasm into cell nucleus and binds to its target gene. Correspondingly, the parameters $r_{9}$ and $r_{10}$ indicate the rates for the association and dissociation of the NF- $\kappa \mathrm{B} \cdot \mathrm{DNA}$ complex, respectively. Given these two rate constants, the number of the NF- $\kappa \mathrm{B} \cdot \mathrm{DNA}$ complex in the system at time $t$ can be changed by solving the following equation.

$$
\frac{d[N F-\kappa B \cdot D N A(t)]}{d t}=r_{9}[N F-\kappa B(t)]-r_{10}[N F-\kappa B \cdot D N A(t)]
$$

While the NF- $\kappa \mathrm{B}$ dissociated from the I $\kappa \mathrm{B} \cdot \mathrm{P} / \mathrm{NF}-\kappa \mathrm{B}$ complex can either bind to DNA or reassociate with unphosphorylated $\mathrm{I} \kappa \mathrm{B}$, the phosphorylated $\mathrm{I} \kappa \mathrm{B}$ that is dissociated from the $\mathrm{I} \kappa \mathrm{B} \cdot \mathrm{P} / \mathrm{NF}-\kappa \mathrm{B}$ complex will be degraded with a rate constant $\mathrm{d} 5$, as described by the following equation.

$$
\frac{d[I \kappa B \cdot P(t)]}{d t}=r_{4}[I \kappa B \cdot P / N F-\kappa B(t)]-d_{5}
$$

Finally, the change of protein $\mathrm{A}_{20}$ is defined by the following equation. 


$$
\frac{d\left[A_{20}(t)\right]}{d t}=\frac{s_{8}[N F-\kappa B \cdot D N A(t-\tau)]}{[N F-\kappa B \cdot D N A(t-\tau)]+K_{8}}-d_{11}
$$

Similarly, its expression is regulated by the NF-kB.DNA complex, as represented the first term on the right-hand side equation (9). The same constant $\tau$ is introduced to describe the time delay between NF- $\mathrm{kB} \cdot \mathrm{DNA}$ formation and $\mathrm{A}_{20}$ expression. The parameters $s_{8}$ and $K_{8}$ in the catalysis of protein synthesis give the maximal rate and saturation coefficient of the reaction as described by Michaelis-Menten kinetics. The second term on the right-hand side of the equation determines the degradation rate of $\mathrm{A}_{20}$.

\section{Numerical algorithm of the hybrid simulation}

The dynamics of the signaling pathway is simulated by two coupled modules. The oligomerization of TRAF scaffold proteins is simulated by a diffusion-reaction algorithm, while the downstream signaling events are simulated by the Gillespie algorithm. In the diffusion-reaction algorithm, we assume the TRAF trimers have bound to the upstream ligand-receptor complexes. Therefore, their movements are confined within the membrane proximal area, which is modeled by a layer of two-dimensional flat surface. Each TRAF protein, together with its upper-bound ligand-receptor complex, is modeled as a single rigid body. In order to capture the basic structural information of the trimeric scaffold protein, the rigid body further contains four connecting groups. The three TRAF$\mathrm{C}$ domains and coiled-coil regions are placed at the center, surrounded by three other groups representing their RING domains. The angle between every two surrounding groups is $120^{\circ}$. Each surrounding group also contains a binding site to mimic the cisinteraction between RING domains.

Given the model representation, the diffusion-reaction simulation is started from an initial configuration, in which a large number of TRAF rigid bodies are randomly distributed within the two-dimensional membrane-proximal region. Each time step in the simulation after the initial configuration is broken down into two scenarios [50]. During the first scenario, all TRAF trimers are chosen by random order to undergo diffusions within the two-dimensional area. The rotations of each trimer are restricted along the surface normal, while the translational movements are along any direction in the plain. 
The periodic boundary condition is applied so that any trimer leaving the $2 \mathrm{D}$ simulation box will enter its opposite side. Following the scenario of molecular diffusions, the binding kinetics of cis-interaction between TRAF trimers is simulation in the second scenario. The association between two TRAF trimers can be triggered if: 1) the distance between any binding sites within these two trimers is below a predetermined cutoff; and 2) their packing angle equals $180^{\circ}$, as shown in Figure 1a. If a cis-interaction is formed between two trimers, they will stop diffusing to facilitate further oligomerization. On the other hand, dissociation of a cis-interaction occurs with a probability that is determined by its binding affinity. After dissociation, two trimers can either reassociate as a geminate recombination if the distance and packing angle between any pair of their binding sites satisfy the association criteria, or diffuse farther away from each other. The new configuration is updated at the end of each simulation time step after both diffusion and reaction scenarios are sequentially performed. Finally, the iteration of above diffusionreaction process will not be terminated until the dynamics of the simulated system reaches equilibrium.

The high-order clusters formed by TRAF scaffold proteins provide a platform to recruit the poly-ubiquitin chains. The downstream signaling processes activated by the poly-ubiquitination are modeled by non-spatial stochastic simulation algorithm. This algorithm was developed by Gillespie in order to study biochemical reactions [51]. Given the initial condition of a signaling network, the populations of each molecular species in the system are propagated in a digitalized and stochastic manner. In detail, within each simulation step, the rates for all reactions are sequentially calculated by the mathematical formulas described in the previous section of the method. A cumulative distribution function is generated by adding up all these rates. A random number is used to select one of these reactions based on the cumulative distribution function. Populations for all species are then updated according to the selected reaction. The simulation moves forward iteratively by above procedure. This Gillespie algorithm has further been coupled with the diffusion-reaction model [52], so that the spatial information of TRAF clustering can be integrated into the downstream signaling processes. Specifically, after the diffusion-reaction simulation, the number of newly formed cis-interactions between TRAF trimers can be obtained. Based on current knowledge about the function of TRAF 
protein in NF- $\mathrm{KB}$ signaling pathway, we assume that poly-ubiquitin chains are recruited at each cis-binding interface of TRAF trimers. Therefore, the number of LUBAC will be simultaneously updated. This new number of LUBAC enters the next step of the Gillespie simulation to guide the activation of IKK. In turn, the outputs from the Gillespie simulation affect the diffusion-reaction simulation as follow. Based on the Gillespie algorithm, the time of the simulation system is moved forward by a specific time interval $\tau$. Given this new time interval, $n$ steps of the diffusion-reaction simulation are carried out to generate a new spatial configuration. The number $n$ is calculated as $\tau / \Delta t$, in which $\Delta \mathrm{t}$ is the length of the diffusion-reaction simulation time step. This calculation is to ensure that the time scale between spatial processes of TRAF clustering and the downstream signaling pathway can be synchronized.

We described a generic framework of NF- $\mathrm{KB}$ signaling network in which some factors have not been simulated before. It is not realistic to derive consistent model parameters from various experimental measurements or previous computational models. As a result, the values of all parameters were chosen on a heuristic basis from the biologically meaningful range, so that the oscillatory nature of the pathway under a persistent ligand stimulation can be qualitatively reproduced within the timescale that is close to the experimental observation. The values of these parameters in the simulation are listed in the supplemental Table S1. The variations in these parameters will not significantly affect the general dynamic patterns of the system. It is more important to recognize how the spatial-temporal dynamics of the signaling system is quantitatively modified by a small perturbation in the parameters space. 


\section{Acknowledgement}

This work was supported by the National Institutes of Health under Grant Numbers R01GM120238 and R01GM122804. The work is also partially supported by a start-up grant from Albert Einstein College of Medicine. Computational support was provided by Albert Einstein College of Medicine High Performance Computing Center.

\section{Author Contributions}

K.D. and Y.W. designed research; K.D. performed research; K.D. and Z.S. analyzed data; K.D. and Y.W. wrote the paper.

Data Availability: Data sharing is not applicable to this article as no datasets were generated or analyzed during the current study.

Competing interests: The authors declare no competing interests. 


\section{References}

1. Gobert, A.P., K.T. Wilson, and C. Martin, Cellular responses to attaching and effacing bacteria: activation and implication of the innate immune system. Arch Immunol Ther Exp (Warsz), 2005. 53(3): p. 234-44.

2. Lacy, P. and J.L. Stow, Cytokine release from innate immune cells: association with diverse membrane trafficking pathways. Blood, 2011. 118(1): p. 9-18.

3. Coussens, L.M. and Z. Werb, Inflammation and cancer. Nature, 2002. 420(6917): p. 8607.

4. Courtois, G. and T.D. Gilmore, Mutations in the NF-kappaB signaling pathway: implications for human disease. Oncogene, 2006. 25(51): p. 6831-43.

5. Kumar, V. and A. Sharma, Neutrophils: Cinderella of innate immune system. Int Immunopharmacol, 2010. 10(11): p. 1325-34.

6. Gilmore, T.D., Introduction to NF-kappaB: players, pathways, perspectives. Oncogene, 2006. 25(51): p. 6680-4.

7. Brasier, A.R., The NF-kappaB regulatory network. Cardiovasc Toxicol, 2006. 6(2): p. 11130.

8. Akira, S. and K. Takeda, Toll-like receptor signalling. Nat Rev Immunol, 2004. 4(7): p. 499-511.

9. Park, H.H., Structure of TRAF Family: Current Understanding of Receptor Recognition. Front Immunol, 2018. 9: p. 1999.

10. Lamothe, B., et al., Site-specific Lys-63-linked tumor necrosis factor receptor-associated factor 6 auto-ubiquitination is a critical determinant of I kappa B kinase activation. J Biol Chem, 2007. 282(6): p. 4102-12.

11. Ghosh, S. and D. Baltimore, Activation in vitro of NF-kappa B by phosphorylation of its inhibitor I kappa B. Nature, 1990. 344(6267): p. 678-82.

12. Sen, R. and D. Baltimore, Inducibility of kappa immunoglobulin enhancer-binding protein Nf-kappa B by a posttranslational mechanism. Cell, 1986. 47(6): p. 921-8.

13. Cebecauer, M., et al., Signalling complexes and clusters: functional advantages and methodological hurdles. Journal of Cell Science, 2010. 123(3): p. 309-320.

14. Hartman, N.C. and J.T. Groves, Signaling clusters in the cell membrane. Current Opinion in Cell Biology, 2011. 23(4): p. 370-376.

15. Napetschnig, J. and H. Wu, Molecular basis of NF-kappaB signaling. Annu Rev Biophys, 2013. 42: p. 443-68.

16. Yin, Q., et al., E2 interaction and dimerization in the crystal structure of TRAF6. Nat Struct Mol Biol, 2009. 16(6): p. 658-66.

17. O'Neill, L.A. and A.G. Bowie, The family of five: TIR-domain-containing adaptors in Tolllike receptor signalling. Nat Rev Immunol, 2007. 7(5): p. 353-64.

18. Xie, P., TRAF molecules in cell signaling and in human diseases. J Mol Signal, 2013. 8(1): p. 7.

19. Middleton, A.J., et al., The activity of TRAF RING homo- and heterodimers is regulated by zinc finger 1. Nat Commun, 2017. 8(1): p. 1788.

20. Cheong, R., A. Hoffmann, and A. Levchenko, Understanding NF-kappaB signaling via mathematical modeling. Mol Syst Biol, 2008. 4: p. 192.

21. Cheong, R., et al., Transient IkappaB kinase activity mediates temporal NF-kappaB dynamics in response to a wide range of tumor necrosis factor-alpha doses. J Biol Chem, 2006. 281(5): p. 2945-50. 
22. Hoffmann, A., et al., The IkappaB-NF-kappaB signaling module: temporal control and selective gene activation. Science, 2002. 298(5596): p. 1241-5.

23. Pogson, M., et al., Introducing spatial information into predictive NF-kappaB modelling-an agent-based approach. PLoS One, 2008. 3(6): p. e2367.

24. Pogson, M., et al., Formal agent-based modelling of intracellular chemical interactions. Biosystems, 2006. 85(1): p. 37-45.

25. Pękalski, J., et al., Spontaneous NF-KB activation by autocrine TNF $\alpha$ signaling: $a$ computational analysis. PLoS One, 2013. 8(11): p. e78887.

26. Turner, D.A., et al., Physiological levels of TNFalpha stimulation induce stochastic dynamics of NF-kappaB responses in single living cells. J Cell Sci, 2010. 123(Pt 16): p. 2834-43.

27. Stiles, Jr. and T.M. Bartol, Monte Carlo methods for simulating realistic synaptic microphysiology using MCell. Computational Neuroscience, 2001: p. 87-127.

28. Andrews, S.S., Smoldyn: particle-based simulation with rule-based modeling, improved molecular interaction and a library interface. Bioinformatics, 2017. 33(5): p. 710-717.

29. Blinov, M.L., et al., Compartmental and Spatial Rule-Based Modeling with Virtual Cell. Biophys J, 2017. 113(7): p. 1365-1372.

30. Michalski, P.J. and L.M. Loew, SpringSaLaD: A Spatial, Particle-Based Biochemical Simulation Platform with Excluded Volume. Biophys J, 2016. 110(3): p. 523-529.

31. Chattaraj, A., M. Youngstrom, and L.M. Loew, The Interplay of Structural and Cellular Biophysics Controls Clustering of Multivalent Molecules. Biophys J, 2019. 116(3): p. 560572.

32. Slepchenko, B.M., et al., Computational cell biology: spatiotemporal simulation of cellular events. Annu Rev Biophys Biomol Struct, 2002. 31: p. 423-41.

33. Slepchenko, B.M., et al., Quantitative cell biology with the Virtual Cell. Trends Cell Biol, 2003. 13(11): p. 570-6.

34. Francke, C., et al., Why the phosphotransferase system of Escherichia coli escapes diffusion limitation. Biophys J, 2003. 85(1): p. 612-22.

35. Hattne, J., D. Fange, and J. Elf, Stochastic reaction-diffusion simulation with MesoRD. Bioinformatics, 2005. 21(12): p. 2923-4.

36. Ander, M., et al., SmartCell, a framework to simulate cellular processes that combines stochastic approximation with diffusion and localisation: analysis of simple networks. Syst Biol (Stevenage), 2004. 1(1): p. 129-38.

37. Rodriguez, J.V., et al., Spatial stochastic modelling of the phosphoenolpyruvatedependent phosphotransferase (PTS) pathway in Escherichia coli. Bioinformatics, 2006. 22(15): p. 1895-901.

38. Andrews, S.S. and D. Bray, Stochastic simulation of chemical reactions with spatial resolution and single molecule detail. Phys Biol, 2004. 1(3-4): p. 137-51.

39. Ridgway, D., et al., Coarse-grained molecular simulation of diffusion and reaction kinetics in a crowded virtual cytoplasm. Biophysical Journal, 2008. 94(10): p. 3748-3759.

40. Frazier, Z. and F. Alber, A Computational Approach to Increase Time Scales in Brownian Dynamics-Based Reaction-Diffusion Modeling. Journal of Computational Biology, 2012. 19(6): p. 606-618.

41. Lalani, A.l., et al., TRAF molecules in inflammation and inflammatory diseases. Curr Pharmacol Rep, 2018. 4(1): p. 64-90.

42. Kim, C.M., et al., Crystal structure of TRAF1 TRAF domain and its implications in the TRAF1-mediated intracellular signaling pathway. Sci Rep, 2016. 6: p. 25526. 
43. Spit, M., E. Rieser, and H. Walczak, Linear ubiquitination at a glance. J Cell Sci, 2019. 132(2).

44. Kearns, J.D., et al., IkappaBepsilon provides negative feedback to control NF-kappaB oscillations, signaling dynamics, and inflammatory gene expression. J Cell Biol, 2006. 173(5): p. 659-64.

45. Zambrano, S., et al., NF-KB oscillations translate into functionally related patterns of gene expression. Elife, 2016. 5: p. e09100.

46. Qian, H., Cooperativity in cellular biochemical processes: noise-enhanced sensitivity, fluctuating enzyme, bistability with nonlinear feedback, and other mechanisms for sigmoidal responses. Annu Rev Biophys, 2012. 41: p. 179-204.

47. Liu, T., et al., NF-KB signaling in inflammation. Signal Transduct Target Ther, 2017. 2: $\mathrm{p}$. 17023-.

48. Nussinov, R., The spatial structure of cell signaling systems. Physical biology, 2013. 10(4): p. 045004-045004.

49. Huang, B., et al., Interrogating the topological robustness of gene regulatory circuits by randomization. PLoS Comput Biol, 2017. 13(3): p. e1005456.

50. Xie, Z.-R., J. Chen, and Y. Wu, A coarse-grained model for the simulations of biomolecular interactions in cellular environments. Journal of Chemical Physics, 2014. 140: p. 054112.

51. Gillespie, D.T., Stochastic simulation of chemical kinetics, in Annual Review of Physical Chemistry. 2007, Annual Reviews: Palo Alto. p. 35-55.

52. Chen, J., Z.R. Xie, and Y. Wu, Elucidating the Functional Roles of Spatial Organization in Cross-Membrane Signal Transduction by a Hybrid Simulation Method. J Comput Biol, 2016. 23(7): p. 566-84. 


\section{Figure Legends}

Figure 1: The dynamics of the NF- $\mathrm{NB}$ signaling pathway is simulated by a computational method consisting of two coupled systems. The assembly of TRAF-based signaling platform at the membrane-proximal region is modeled by a rigid-body-based diffusionreaction algorithm, as shown in (a). We assume that each TRAF trimer has bound to its upstream ligand-receptor complex and therefore is modeled as a single rigid body which movements are confined within the two-dimensional membrane proximal area. The formation of a cis-interaction between two TRAF trimers thus facilitates the assembly of linear ubiquitin chain assembly complex (LUBAC). The LUBAC further provide the scaffold to enable the activation of downstream signaling pathway, which diagram is sketched in (b). The dynamics of the signaling network is simulated by Gillespie algorithm. Finally, the diffusion-reaction and Gillespie algorithms are synchronized under a multiscale simulation framework, as described in the Method.

Figure 2: The initial configuration of the diffusion-reaction simulation is shown in (a). Some representative snapshots along the simulation trajectory were selected in (b) and (c). The configuration that the system reached equilibrium is further shown in (d). In (e), we plotted the number of cis-interactions between TRAF trimers observed in the system along with the simulation time steps. The kinetic profiles of molecular components in the downstream signaling network were further plotted as functions of simulation time steps. These profiles are: the numbers of NF- $\kappa$ B molecules which bind to I $\kappa \mathrm{B}$ and DNA as shown by the blue and orange curves in (f); and the total number of free IкB in the system as shown by the red curve in $(\mathbf{g})$.

Figure 3: Two simulation scenarios were designed to elucidate the functional importance of TRAF clustering in regulation of downstream signaling pathway. The TRAF trimers in the first scenario contain three binding sites and can simultaneously form cis-interactions with three structural neighbors, while in a control system only one binding site was assigned to each TRAF protein. The numbers of cis-interactions formed along the simulations in these two scenarios are plotted in (a). The distributions of fluctuations in the number cis-interactions are shown as histograms in (b). The final configurations at the end of two simulation systems are compared with each other in (c) and (d). Finally, 
the outputs from the downstream signaling networks of these two systems, e.g. the

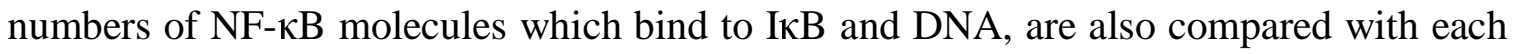
other in (e) and (f), respectively.

Figure 4: In order to explore the energetic impacts of the cis-interaction between TRAF trimers on spatial-temporal dynamics of the system. Three scenarios with the weak, moderate and strong binding affinities of these cis-interactions were tested separately. The numbers of cis-interactions formed in these three scenarios are plotted in (a) as a function of simulation time steps, while the numbers of free $\mathrm{I} \kappa \mathrm{B}$ in the system are shown in (b). More systematical tests were further carried out in which we changed the binding affinities of cis-interaction from $-6 \mathrm{kT}$ to $-15 \mathrm{kT}$ with an interval of $1 \mathrm{kT}$. The average numbers of oscillation periods were derived from the multiple trajectories of these system, and were plotted as histogram in (c) as a function of cis-binding affinity.

Figure 5: To illustrate the relation between the spatial properties of TRAF clusters and the strength of their cis-interactions, the final configurations from the systems which binding affinities equal $-7 \mathrm{kT}$ and $-8 \mathrm{kT}$ are plotted in (a) and (b). These two plots show that the clustering can generate very different spatial pattern with only a slight change in the binding affinity. On the other hand, the final configurations from the systems which binding affinities equal $-13 \mathrm{kT}$ and $-15 \mathrm{kT}$ are plotted in (c) and (d), respectively. These two plots suggest that systems with stronger binding affinity contain more clusters with smaller sizes. 


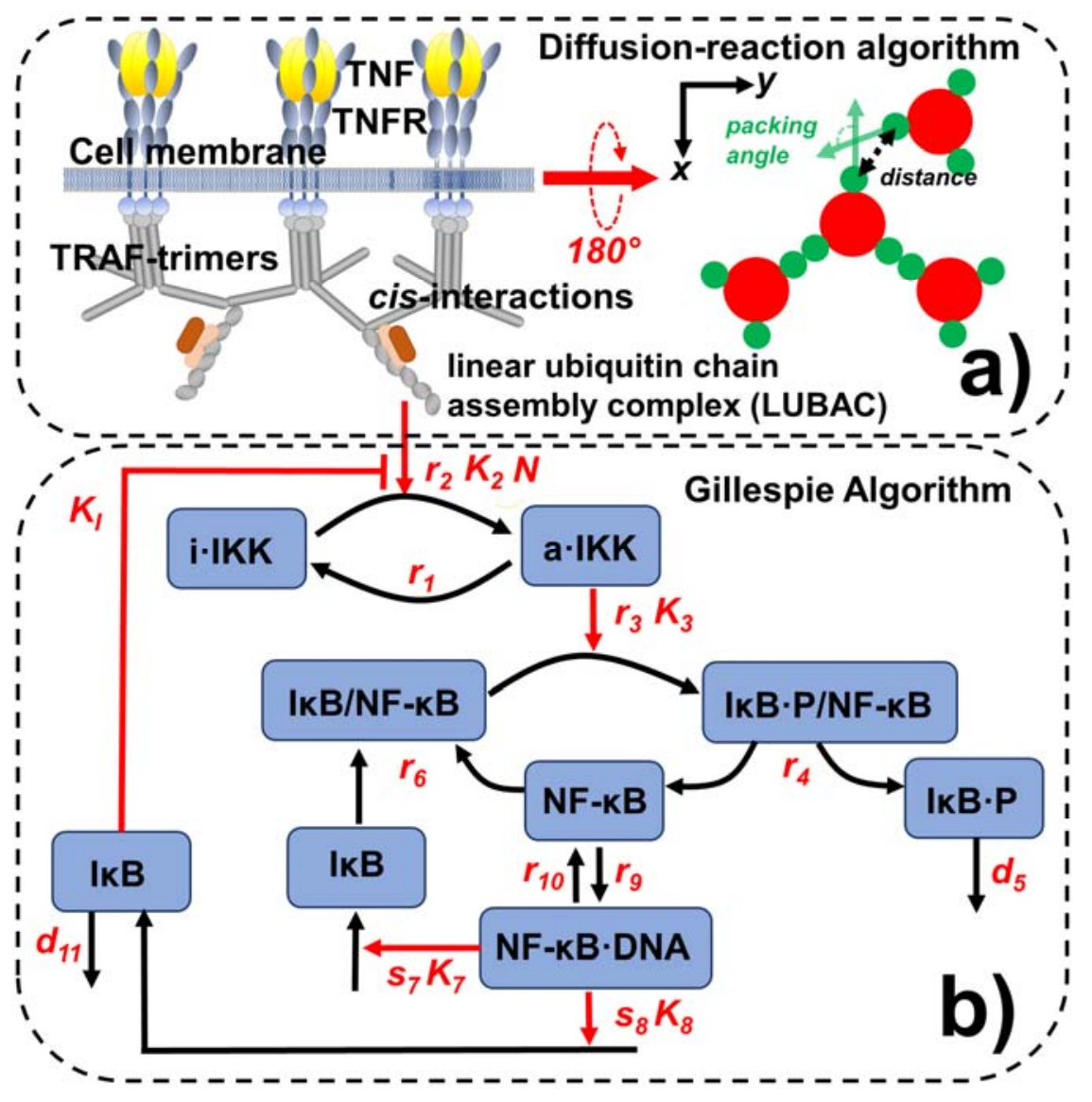

Figure 1 
bioRxiv preprint doi: https://doi.org/10.1101/2020.11.11.378331; this version posted November 11,2020 . The copyright holder for this preprint (which was not certified by peer review) is the author/funder. All rights reserved. No reuse allowed without permission.

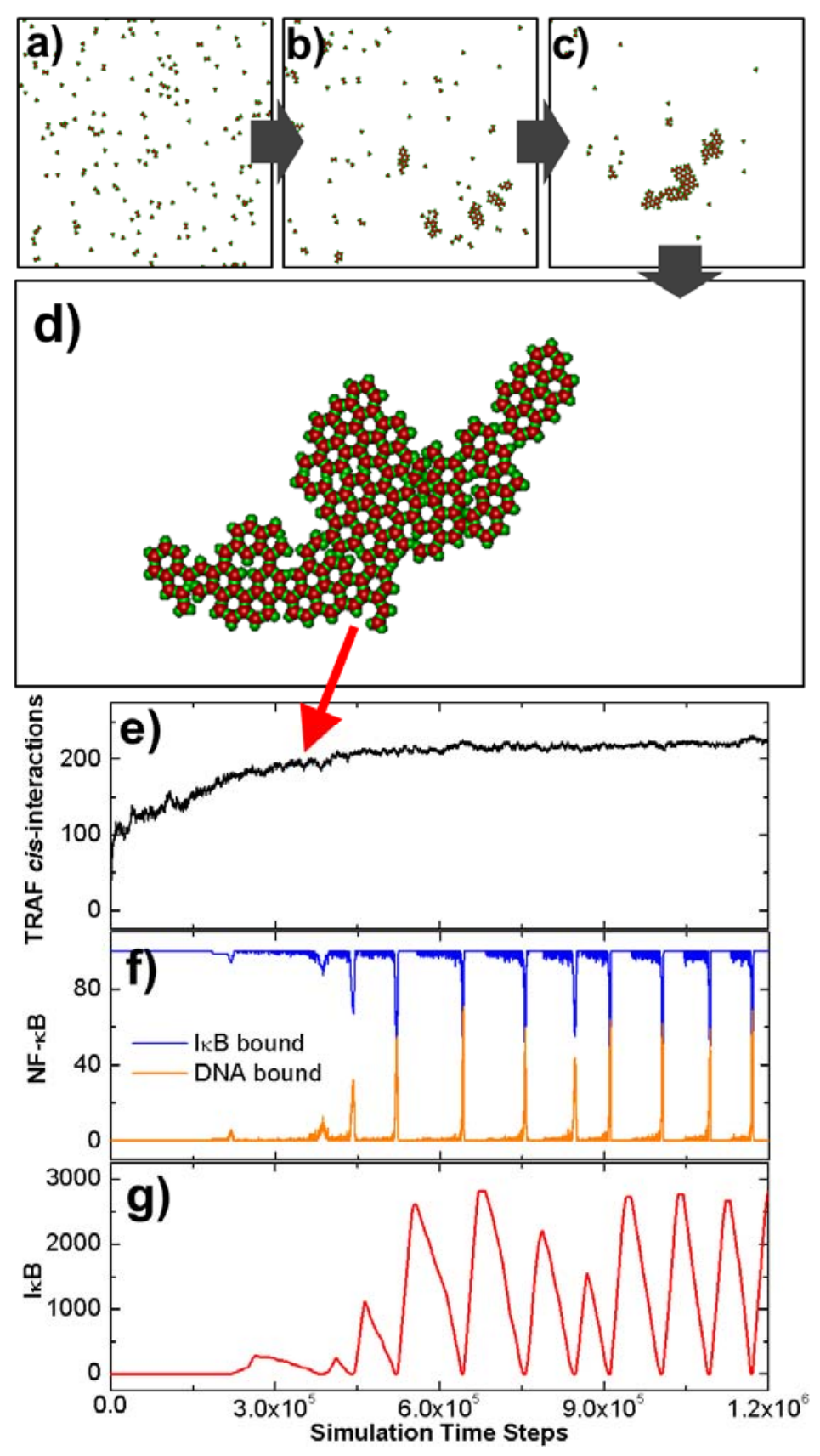

Figure 2 

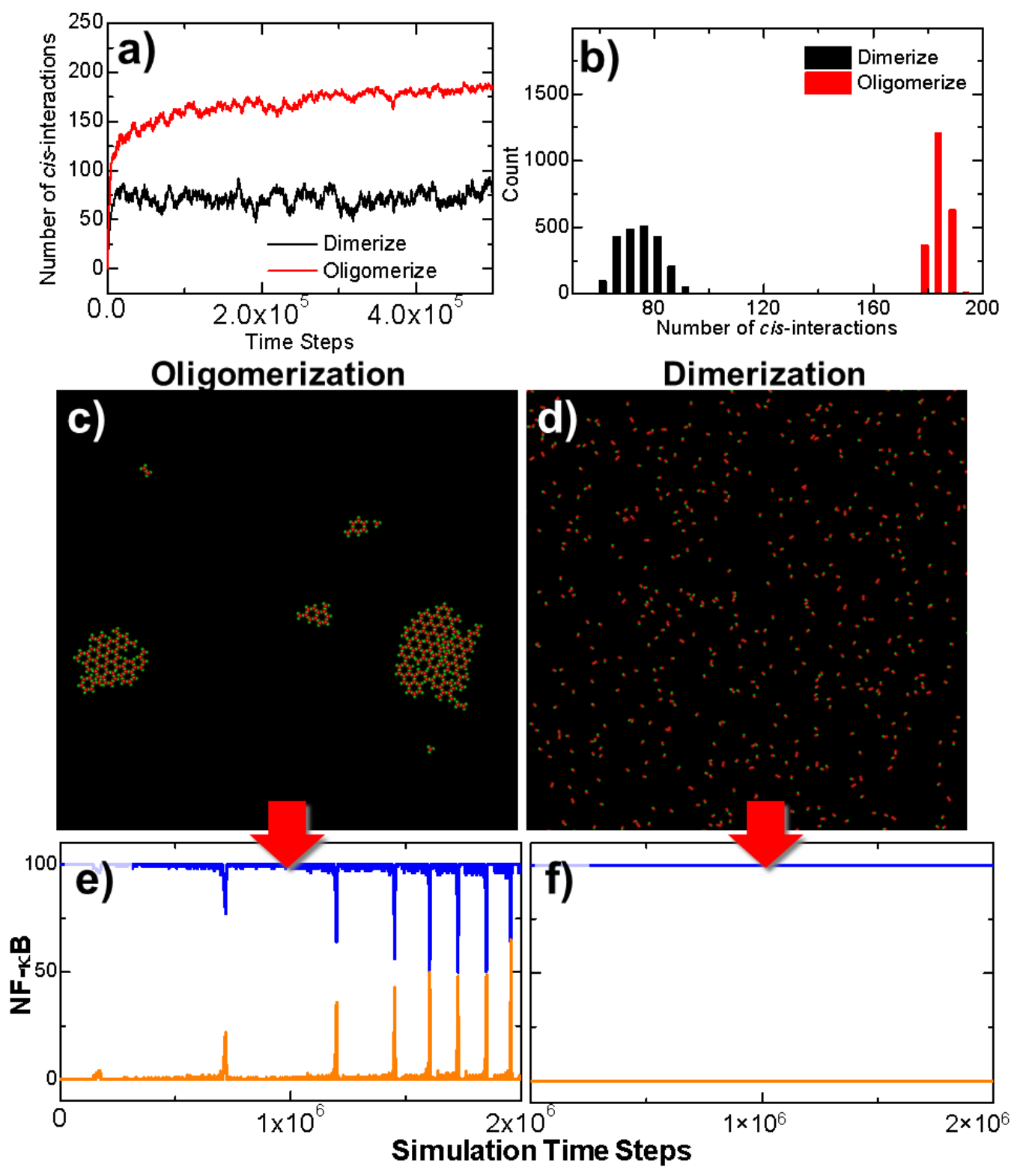

Figure 3 

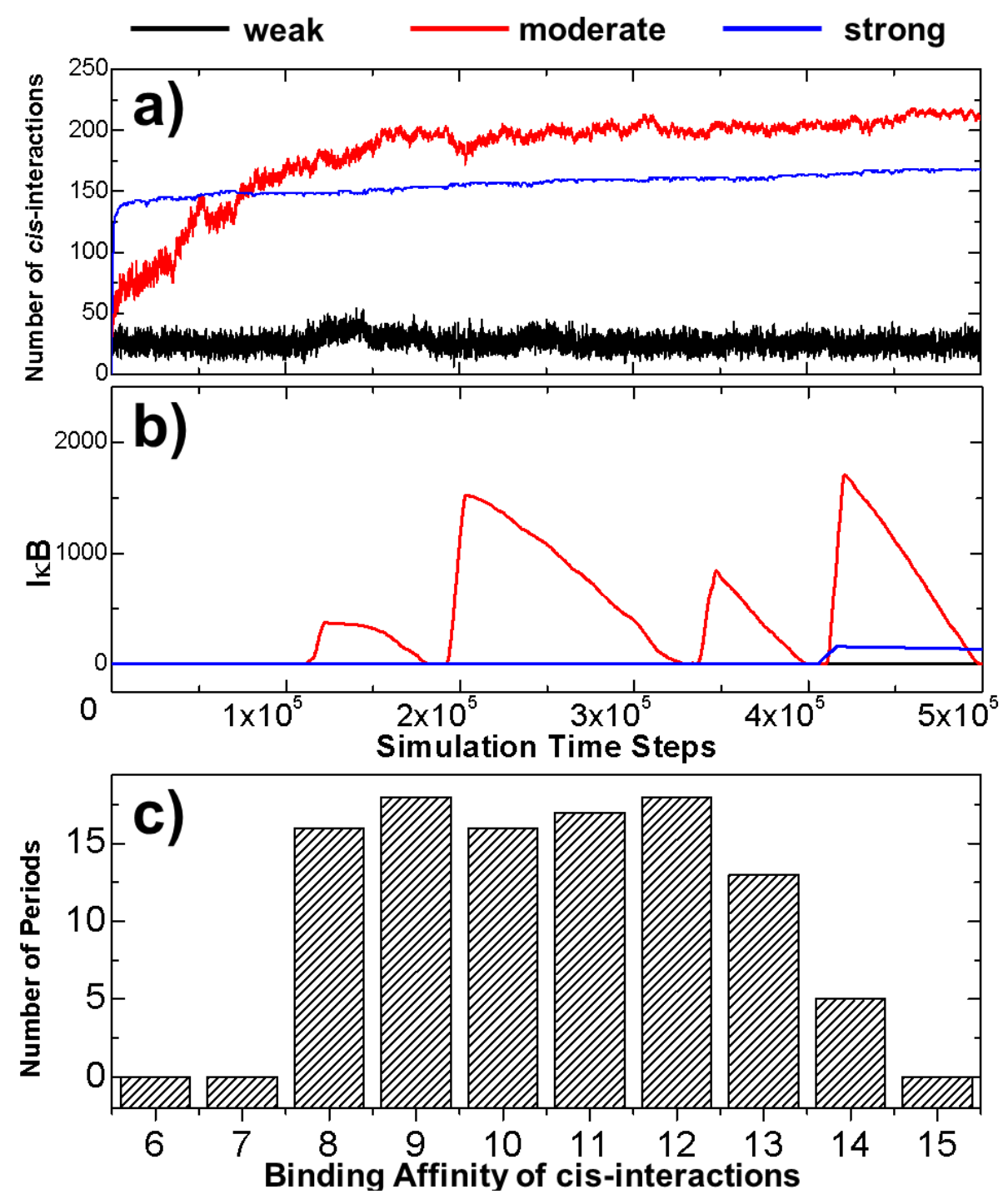

Figure 4 

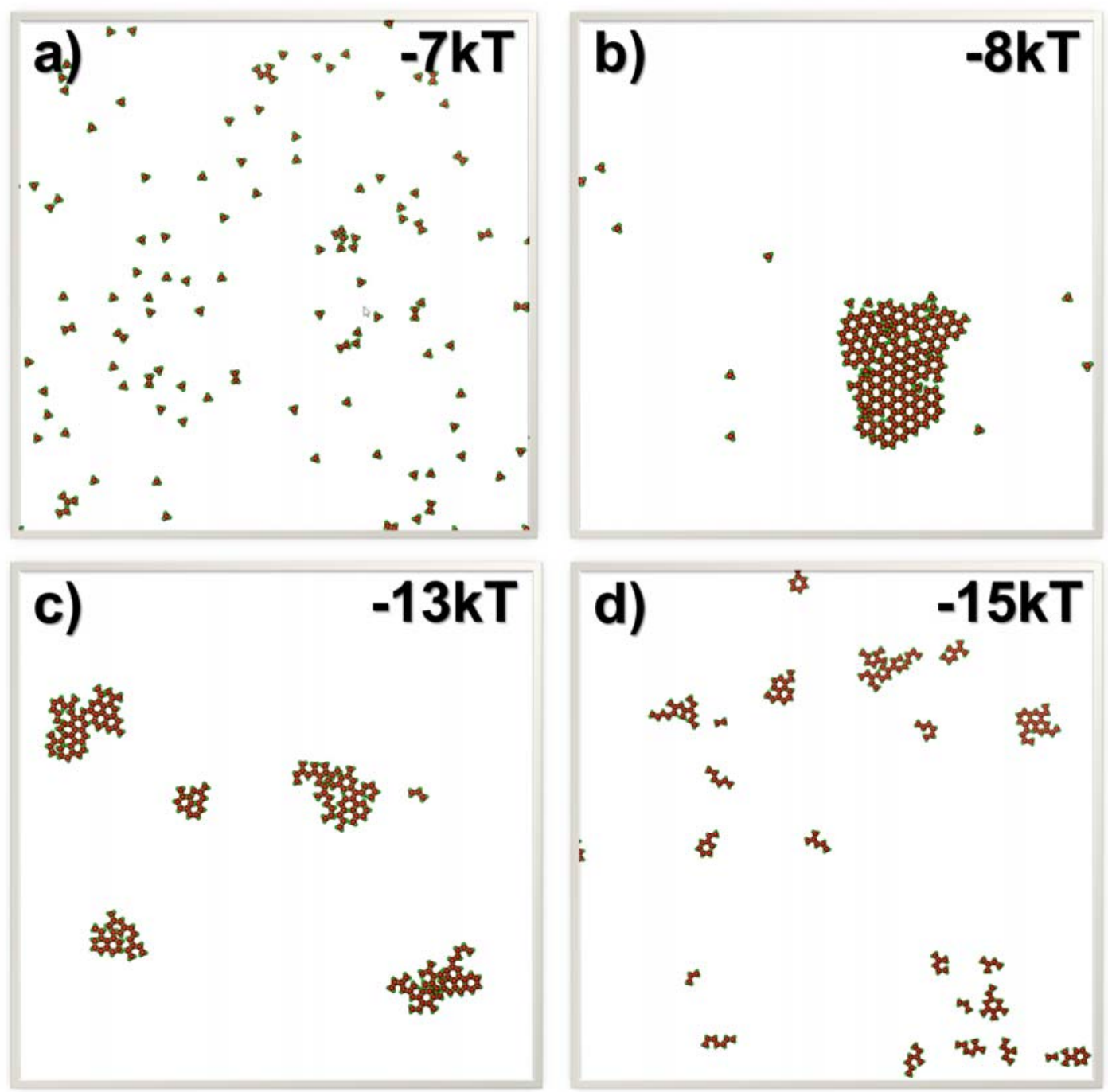

Figure 5 
bioRxiv preprint doi: https://doi.org/10.1101/2020.11.11.378331; this version posted November 11, 2020. The copyright holder for this preprint (which was not certified by peer review) is the author/funder. All rights reserved. No reuse allowed without permission.

2020-09-26

Simulation of spatial Organization in NF-KB Pathway

\begin{tabular}{|c|c|c|c|c|c|}
\hline Index & Description & Mathematical Representation & Parameter & Value & Remark \\
\hline 1 & $\begin{array}{l}\text { Deactivation of } \\
\text { IKK }\end{array}$ & $r_{1}[a \cdot I K K(t)]$ & $r_{l}$ & $0.2 \mathrm{~s}^{-1}$ & $\begin{array}{l}\text { Rate of } \\
\text { transition }\end{array}$ \\
\hline \multirow{4}{*}{2} & \multirow{4}{*}{ Activation of IKK } & \multirow{4}{*}{$r_{2}[i \cdot \operatorname{IKK}(t)] \times \frac{[\operatorname{LUBAC}(t)]^{N}}{[\operatorname{LUBAC}(t)]^{N}+\left[K_{2} \times\left(1+\left[A_{20}(t)\right] / K_{I}\right)\right]^{N}}$} & $r_{2}$ & $0.4 \mathrm{~s}^{-1}$ & $\begin{array}{l}\text { Maximal rate } \\
\text { of catalysis }\end{array}$ \\
\hline & & & $K_{2}$ & $240 \mathrm{nM}$ & $\begin{array}{l}\text { Saturation } \\
\text { coefficient of } \\
\text { catalysis }\end{array}$ \\
\hline & & & $K_{I}$ & $10 \mathrm{nM}$ & $\begin{array}{l}\text { Inhibition } \\
\text { coefficient of } \\
\text { catalysis }\end{array}$ \\
\hline & & & $N$ & 25 & $\begin{array}{c}\text { Hill } \\
\text { coefficient }\end{array}$ \\
\hline \multirow[b]{2}{*}{3} & \multirow{2}{*}{$\begin{array}{l}\text { Phosphorylation } \\
\text { of I } \mathrm{B} B\end{array}$} & \multirow{2}{*}{$\frac{r_{3}[a \cdot I K K(t)][I \kappa B / N F-\kappa B(t)]}{[I \kappa B / N F-\kappa B(t)]+K_{3}}$} & $r_{3}$ & $0.08 \mathrm{~s}^{-1}$ & $\begin{array}{c}\text { Maximal rate } \\
\text { of catalysis }\end{array}$ \\
\hline & & & $K_{3}$ & $500 \mathrm{nM}$ & $\begin{array}{l}\text { Saturation } \\
\text { coefficient of } \\
\text { catalysis }\end{array}$ \\
\hline 4 & 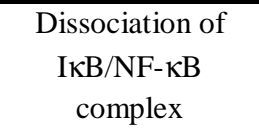 & $r_{4}[I \kappa B \cdot P / N F-\kappa B(t)]$ & $r_{4}$ & $0.4 \mathrm{~s}^{-1}$ & $\begin{array}{l}\text { Dissociation } \\
\quad \text { rate }\end{array}$ \\
\hline 5 & $\begin{array}{l}\text { Degradation of } \\
\text { IкB }\end{array}$ & $d_{5}$ & $d_{5}$ & $0.8 \mathrm{nMs}^{-1}$ & $\begin{array}{l}\text { Degradation } \\
\text { rate }\end{array}$ \\
\hline 6 & $\begin{array}{l}\text { Association of } \\
\text { I } \kappa / \mathrm{NF}-\kappa \mathrm{B} \\
\text { complex }\end{array}$ & $r_{6}[I \kappa B(t)][N F-\kappa B(t)]$ & $r_{6}$ & $\begin{array}{l}0.008 \mathrm{nM}^{-} \\
{ }^{1} \mathrm{~s}^{-1}\end{array}$ & $\begin{array}{l}\text { Association } \\
\text { rate }\end{array}$ \\
\hline \multirow[b]{2}{*}{7} & \multirow[b]{2}{*}{ Synthesis of IкB } & $s_{7}[N F-\kappa B \cdot D N A(t-\tau)]$ & $s_{7}$ & $0.8 \mathrm{~s}^{-1}$ & $\begin{array}{l}\text { Maximal rate } \\
\text { of synthesis }\end{array}$ \\
\hline & & {$[N F-\kappa B \cdot D N A(t-\tau)]+K_{7}$} & $K_{7}$ & $50 \mathrm{nM}$ & $\begin{array}{c}\text { Saturation } \\
\text { coefficient of } \\
\text { synthesis }\end{array}$ \\
\hline \multirow[b]{2}{*}{8} & \multirow[b]{2}{*}{ Synthesis of $A_{20}$} & $s_{8}[N F-\kappa B \cdot D N A(t-\tau)]$ & $s_{8}$ & $0.4 \mathrm{~s}^{-1}$ & $\begin{array}{l}\text { Maximal rate } \\
\text { of synthesis }\end{array}$ \\
\hline & & {$[N F-\kappa B \cdot D N A(t-\tau)]+K_{8}$} & $K_{8}$ & $100 \mathrm{nM}$ & $\begin{array}{c}\text { Saturation } \\
\text { coefficient of } \\
\text { synthesis }\end{array}$ \\
\hline 9 & $\begin{array}{c}\text { Association of } \\
\text { NF- } \kappa B \text { with DNA }\end{array}$ & $r_{9}[N F-\kappa B(t)]$ & $r_{9}$ & $0.8 \mathrm{~s}^{-1}$ & Transition rate \\
\hline 10 & $\begin{array}{c}\text { Dissociation of } \\
\mathrm{NF}-\kappa \mathrm{B} \text { from DNA }\end{array}$ & $r_{10}[N F-\kappa B \cdot D N A(t)]$ & $r_{10}$ & $0.08 \mathrm{~s}^{-1}$ & Transition rate \\
\hline 11 & $\begin{array}{c}\text { Degradation of } \\
\mathrm{A}_{20}\end{array}$ & $d_{11}$ & $d_{11}$ & $0.1 \mathrm{nMs}^{-1}$ & $\begin{array}{l}\text { Degradation } \\
\text { rate }\end{array}$ \\
\hline
\end{tabular}

Table 1: the information about the simulation parameters in the signaling network 